Polymer Journal, Vol. 5, No. 1, pp 91-96 1973

\title{
Stress Relaxation of Polymer Solutions under Large Strain*
}

\author{
Yoshiyuki Einaga, ${ }^{* *}$ Kunihiro Osaki, and Michio Kurata \\ Institute for Chemical Reseacrh, Kyoto University \\ Uji, Kyoto 611, Japan. \\ Shin-ichi KImURA, ${ }^{* * *}$ Nobuhiro YAmada, ${ }^{* * * *}$ and Mikio TAmURA \\ Department of Industrial Chemistry, Kyoto University \\ Sakyo, Kyoto 606, Japan.
}

(Received February 26, 1973)

\begin{abstract}
Shear relaxation moduli $G(t, s)$ were measured for various strains $s$ with a relaxometer of the cone-plate type for concentrated solutions of polystyrene in chlorinated biphenyl. The strain was varied from 0.34 to 25 shear units. The timetemperature reduction method was applicable to the shear-dependent relaxation moduli over the temperature range studied $\left(20-50^{\circ} \mathrm{C}\right)$ and the shift factor $a_{\mathrm{T}}$ was independent of $s$. When $s$ was very large, $\log G(t, s)$ decreased very rapidly with increasing time in two regions of $t$ (two-step relaxation) while $\log G(t, s)$ for small $s$ dropped rapidly only at long times (one-step relaxation). The relative rate of decrease of $G(t, s)$ with increasing $s$ was independent of $t$ when $t$ was larger than a certain time, $\tau_{k}$. The maximum relaxation time $\tau_{\mathrm{m}}$ was independent of $s$ while the corresponding strength of relaxation $G_{\mathrm{m}}$ was proportional to $I_{D}^{-0.83}$ when $s$ was large. Here $I_{D}$ is the first invariant of strain.

KEY WORDS Stress Relaxation / Relaxation Time / Large Strain /

Nonlinear Behavior / Polystyrene / Solution / Entanglement /
\end{abstract}

Nonlinear viscoelasticity of polymer concentrates has recently acquired increasing interest. Stress components have been investigated for various types of flow: steady shear, start and cessation of steady shear, oscillatory shear of various amplitudes, superposition of oscillatory shear on steady shear, elongational flow, and so on. ${ }^{1}$ In order to describe the observed stress components for any type of deformation, constitutive equations or equations of rheological state have been constructed by many investigators, and some of the equations attained considerable success in describing the stress components observed so far. ${ }^{1}$ However, most of the equations require the deformation history

\footnotetext{
* Request for reprints should be addressed to M. Kurata.

** Present address: Department of Polymer Science, Osaka University, Toyonaka.

*** Present address: Japan Synthetic Rubber Co., Ltd., Kawajiricho, Yokkaichi.

**** Present address: Nippon Tokushu Kento, Ltd., Kisshoin, Minami, Kyoto.
}

to be continuous as a function of time and they can not be applied to discontinuous deformation without large modifications. The examples of deformation mentioned above are all of a continuous nature.

On the other hand, an important type of deformation of a discontinuous nature has been used in the field of linear viscoelasticity in relation to the stress relaxation or the measurement of the relaxation modulus. ${ }^{2}$ In the measurement of the relaxation modulus, a certain amount of strain $s$ is imposed on the sample at a certain time, $t=0$, and is kept constant thereafter. The relaxation modulus $G(t)$ is defined as the ratio of the stress supporting the constant strain to the strain in the case when $s$ is so small that the ratio is independent of $s$. The relaxation modulus is one of the simplest functions in linear viscoelasticity and may be very convenient in discussions of relaxation mechanisms of long relaxation times. ${ }^{2}$

The relaxation modulus under large strain $G(t, s)$ may be defined as the ratio of the stress 
to the strain and it may depend on the amount of strain $s$. Measurements of strain-dependent relaxation moduli were reported by Zapas and Craft for elongational deformation of polyisobutylene and plasticized poly(vinyl chloride). ${ }^{3}$ The results were described with a simple constitutive equation of an integral type. The elongation ratios employed were not very large $(<2)$, although they were sufficiently large to affect the relaxation moduli. A very large strain may be attained in simple shear with the use of torsional deformation of the sample in a coneand-plate sample holder, in which the overall shape of the sample does not change even in the case of a very large deformation. As reported in previous papers, we have designed an apparatus for measurements of the relaxation modulus under large strain and have observed that the relaxation modulus is strongly affected by the amount of strain. ${ }^{4,5}$ It may be an interesting project to assess current constitutive equations on the basis of these results. However, we will postpone discussions concerning constitutive equations and try to examine the details of the effect of various strains on the relaxation modulus for polymer concentrates.

\section{EXPERIMENTAL}

Two solutions of polystyrene in Aroclor 1248 were used as samples. The polystyrene was the standard sample $14 \mathrm{a}$ of molecular weight $1.80 \times 10^{6}$ from Pressure Chemical Company. Aroclor 1248 is a mixture of partially chlorinated biphenyls from Monsanto Chemical Company. The density and the viscosity of Aroclor 1248 and the method of preparation of solutions have been previously reported. ${ }^{6,7}$ The concentrations of the solutions were 20 and $22.5 \%$ respectively. The solutions may be classified as concentrated solutions of a polymer of high molecular weight, in which temporary networks of polymer chains due to entanglement coupling are well developed and are of primary importance in relaxation processes of linear viscoelasticity.

An apparatus of a cone-and-plate type was employed for measurements of strain-dependent relaxation moduli. Details of the apparatus were described before. ${ }^{4}$ Measurements were performed in the temperature region of $10-50^{\circ} \mathrm{C}$ for shear strains of $0.341-25.6$ shear units.

\section{RESULTS AND DISCUSSIONS}

\section{Temperature Dependence of Relaxation Modulus}

The effect of varying temperature is examined in Figure 1 for relaxation moduli $G(t, s)$ obtained at various amounts of strain $s$ for the 22.5-\% solution of polystyrene. In this figure reduced relaxation moduli $G_{p}(t, s)=G(t, s) \rho_{0} T_{0} / \rho T$ for various temperatures are plotted against reduced time $t / a_{\mathrm{T}}$, where $T$ is the absolute temperature, $\rho$ is the density of the solution, and the subscript 0 indicates the reference temperature, $33.5^{\circ} \mathrm{C}$ in this case. The shift factor $a_{\mathrm{T}}$ was determined empirically for each temperature so that the best superposition of the data at the temperature on those of the reference temperature is attained. In the case of linear viscoelasticity, this method of reduced plot is of course well known as the method of reduced variables and gives a single composite curve for the relaxation modulus or other viscoelastic functions obtained at various temperatures for many polymeric systems. ${ }^{2}$ The method was successfully applied to the creep compliance of polystyrene solutions in chlorinated biphenyl. ${ }^{7}$ It may be obvious in Figure 1 that

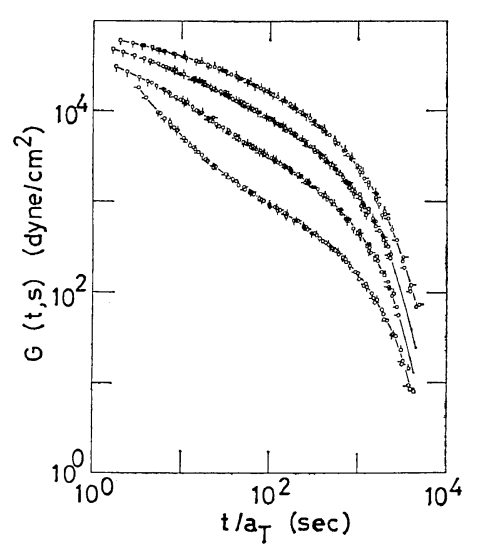

Figure 1. Strain-dependent relaxation moduli $G_{p}(t, s)$ plotted against reduced time $t / a_{\mathrm{T}}$ for 22.5$\%$ solution of polystyrene in Aroclor 1248. Reference temperature is $33.5^{\circ}$. Various directions of pips represent various temperatures: pip right, $50^{\circ}$; succesive $90^{\circ}$ rotations anticlockwise correspond to $40^{\circ}, 33.5^{\circ}$, and $22.5^{\circ}$, respectively. Amounts of strain are $1.87,3.34,6.68$, and 13.4 shear units from top to bottom, respectively. 


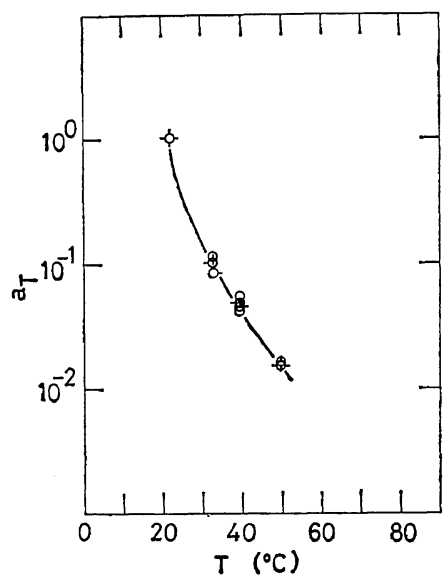

Figure 2. Shift factor $a_{\mathrm{T}}$ plotted against temperature $T$ for strain-dependent relaxation modulus of 22.5-\% polystyrene solution in Aroclor 1248. Various directions of pips represent amounts of shear strain: pip up, 1.87 shear unit; successive $90^{\circ}$ rotations clockwise correspond to $3.34,6.68$, and 13.4 shear units, respectively.

strain-dependent relaxation moduli for various temperatures construct a single composite curve for each amount of strain. The shift factor $a_{T}$ obtained above is plotted against the temperature in Figure 2. It decreases very rapidly with increasing temperature, but it is not affected by the variation of the strain $s$.

The results may be summarized as follows. The time-temperature reduction method is applicable to the relaxation modulus, under large strain, of the polystyrene solution and the shift factor is the same as obtained in the case of small strain. It may be recalled here that the shift factor represents the temperature dependence of the segmental friction coefficient $\zeta$ in the linear viscoelasticity of polymeric systems. ${ }^{2}$ Then the result given above seems to imply that $\zeta$ defined in linear viscoelasticity may also represent a frictional property connected with relaxation mechanisms under large strain.

\section{Master Curves for Relaxation Moduli}

Figure 3 shows the relaxation moduli $G_{p}(t, s)$ reduced to $30^{\circ}$ for the $20-\%$ solution of polystyrene in Aroclor 1248. The strain $s$ was varied from 0.341 to 25.6 shear units. It is seen that $\boldsymbol{G}_{p}(t, s)$ is approximately independent of the $s$ value when $s$ is very small $(s<0.68)$. Thus the

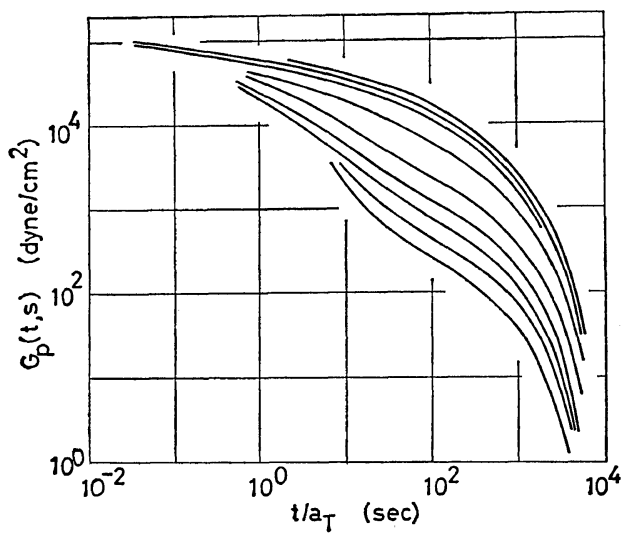

Figure 3. Strain-dependent relaxation moduli $G_{p}(t, s)$ reduced to $30^{\circ}$ for $20-\%$ polystyrene solution in Aroclor 1248. Shear strains $s$ are 0.34 and 0.68 for the first curve at top, then 1.09, 1.71, $3.41,6.83,10.2,13.7,18.8$, and 25.6 for the remaining eight curves, respectively, from top to bottom.

relaxation modulus in this range of strain is approximately equal to the relaxation modulus of linear viscoelasticity. When $s$ is very small, the shape of the curve of $\log G_{p}(t, s)$ as a function of $\log t / a_{\mathrm{T}}$ is in fact similar to that of $\log G(t)$ reported for amorphous polymers in the range of plateau and terminal zones on the time scale. $^{2}$

As the strain increases, $G_{p}(t, s)$ decreases over the whole range of times investigated. However, the relative amount of decrease of $G_{p}(t, s)$ depends on time $t / a_{T}$; it is small in the short time range and increases with time. As has been reported, the relative decrease of $G_{p}(t, s)$ with increasing $s$ becomes independent of time for times longer than a certain time, $\tau_{k}$, so that $\log G_{p}(t, s)$ can be superimposed on $\log G_{p}(t)$ by vertical shift in the plot of $\log G_{p}(t, s) v s$. $\log t / a_{\mathrm{T}}{ }^{5} \quad$ As the result of the different rates of decrease of $G_{p}(t, s)$ with increasing strain in the different time ranges, the shape of the relaxation curves in Figure 3 varies as $s$ increases; $\log G_{p}(t, s)$ for large $s$ decreases very rapidly with increasing $t / a_{\mathrm{T}}$ in two regions of time (two-step relaxation) as compared with that for small $s$ which drops rapidly only at very long times (one-step relaxation).

In order to examine the change of shape in more detail, we will expand the relaxation 
modulus in terms of exponentials of time, namely,

$$
G(t, s)=\sum_{p} G_{p} \mathrm{e}^{-t / \tau_{p}}
$$

where $G_{p}$ and $\tau_{p}$ may be functions of $s$. In the case of linear viscoelasticity, $G_{p}$ represents the strength of the $p$-th relaxation mode of relaxation time $\tau_{p}$. In the case of large $s$ values, the significance of these quantities is still to be clarified, although there is no mathematical difficulty in expanding $G(t, s)$ in the form of eq 1. Following the method of linear viscoelasticity, one may write eq 1 in terms of a continuous function $H(\tau, s)$ as

$$
\boldsymbol{G}(t, s)=\int_{-\infty}^{\infty} H(\tau, s) \mathrm{e}^{-t / \tau} \mathrm{d} \ln \tau
$$

where $H(\tau, s)$ reduces to the distribution function of relaxation time $H(\tau)$ at the limit of $s \rightarrow 0$. When $s$ is large, eq 2 is employed only to investigate the property of $G_{p}$ in eq 1 . It might be noted that functions $G(t, s)$ and $H(\tau, s)$ have been employed in a simple constitutive equation discussed by Yamamoto. ${ }^{8}$

Function $H(\tau, s)$

The function $H(\tau, s)$ was evaluated from the relaxation modulus of Figure 3 for each $s$ value with the approximation method of Ferry and Williams. ${ }^{9}$ Although this method is supposed to be applicable to the relaxation modulus of linear viscoelasticity, it may be employed for evaluation of $H(\tau, s)$ of eq 2 due to the similarity of eq 2 to the expression of the relaxation modulus of linear viscoelasticity. The result is shown in Figure 4, where $H(\tau, s)$ is plotted against $\tau$ for a few selected values of $s$, as indicated. In this figure $\log H(\tau, s)$ as a function of $\log \tau$ for the smallest $s$ value is similar to the relaxation spectrum for amorphous polymers: i.e., $\log H(\tau, s)$ is approximately independent of $\tau$ in the short time range (the plateau region), begins to decrease gradually as $\tau$ increases, and decreases very rapidly in the range of very long $\tau$ (the flow region). ${ }^{2}$ In order to describe $H(\tau, s)$ for large $s$ values, it may be convenient to define $\tau_{l}$ and $\tau_{k}$ and to define three regions on the time scale as indicated in Figure 4. In region 1 , the short time scale $\left(\tau<\tau_{l}\right), H(\tau, s)$ is not much affected by the variation of strain $s$. In

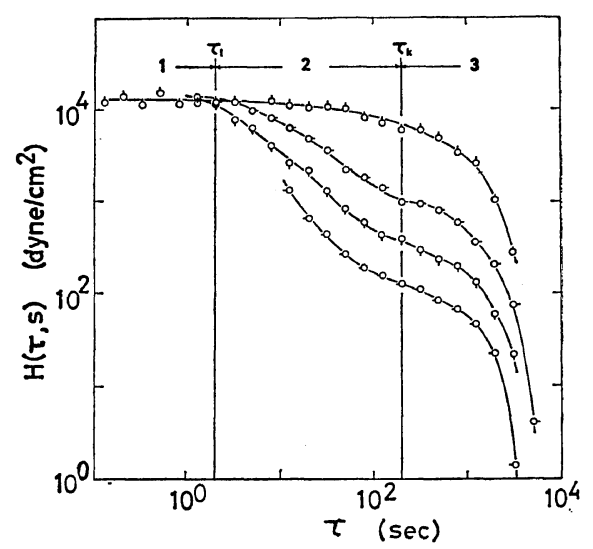

Figure 4. Function $H(\tau, s)$ plotted againts $\tau$ for 20-\% solution of polystyrene in Aroclor 1248. Shear strains $s$ are 1.09, 6.83, 13.7, and 25.6 shear units from top to bottom, respectively. For $\tau \imath$ and $\tau_{k}$ and arrowed regions, see text.

region 3 , the long time scale $\left(\tau<\tau_{k}\right)$, the effect of varying $s$ on $H(\tau, s)$ is the largest and $H(\tau, s)$ decreases as $s$ increases. The way $H(\tau, s)$ decreases is such that $\log H(\tau, s)$ shifts in parallel with the ordinate by a constant amount independent of $\tau$ for each $s$ over region 3 of the time scale. In region 2 , the intermediate time scale $\left(\tau_{l}<\tau<\tau_{k}\right)$, the rate of decrease of $H(\tau, s)$ with increasing $s$ depends on $\tau ; H(\tau, s)$ decreases more rapidly at longer $\tau$. In terms of the discrete expression of eq 1 , this result implies that the strengths $\boldsymbol{G}_{p}$ for the longest few $\tau_{p}$ decrease by a factor independent of $\tau_{p}$ as $s$ increases while $G_{p}$ for very short $\tau_{p}$ are not affected by the variation of $s$.

In describing the effect of varying strain on $H(\tau, s)$, it may be sufficient to specify $\tau_{l}, \tau_{k}$, and the rate of decrease of $H(\tau, s)$ in region 3 . The first parameter $\tau_{l}$ seems to be independent of $s$ although the data in the short time range may be insufficient for a definite conclusion. The second parameter $\tau_{k}$ is also approximately independent of $s$ in Figure 4. This result is in agreement with the comment on $\tau_{k}$ given in a previous paper. ${ }^{5}$ As the measure of the rate of decrease of $H(\tau, s)$ in the long time range, we may take the ratio $G(t) / G(t, s)$ as a function of $s$ and recall that it is proportional to $I_{D}{ }^{0.83}$ when $s$ is very large, where $I_{D}=s^{2}+3$ is the first invariant of the strain tensor. ${ }^{5}$ Here we will 
investigate the effect of varying strain on $G_{p}$ for long $\tau_{p}$ from a different viewpoint. The results for $s$ values smaller than reported are also included.

\section{The Longest Relaxation Time}

The contribution of the term with the longest relaxation time $\tau_{\mathrm{m}}$ to $G(t, s)$ in eq 1 may be evaluated by the method called procedure $X$ if $\tau_{\mathrm{m}}$ is much longer than the second longest relaxation time and the corresponding strength $G_{\mathrm{m}}$ is not very small. ${ }^{10}$ Then $G_{\mathrm{m}} \exp \left(-t / \tau_{\mathrm{m}}\right)$ is much larger than the rest of the terms of eq 1 and $G(t, s)$ is well approximated by this term at long times. Therefore the plot of $\log G(t, s) v s$. $t$ will give a straight line, with intercept $\log G_{\mathrm{m}}$ and slope $-1 / 2.303 \tau_{\mathrm{m}}$, when $t$ is large. An example of this plot is shown in Figure 5 for a few selected values of $s$ for the relaxation modulus of Figure 3. It may be obvious that long fractions of straight lines are obtained for all the $s$ values when $t$ is large and that the straight lines are approximately parallel with each other.

The values of $G_{\mathrm{m}}$ and $\tau_{\mathrm{m}}$ as evaluated with procedure $X$ for the data of Figure 3 are plotted against $I_{D} / 3$ in Figure 6 . The choice of the variable for the abscissa is only for aesthetic reasons. Apparently $\tau_{\mathrm{m}}$ does not depend on the strain $s$ very much. It does decrease slightly when $s$ becomes very large $(s>10)$ and this decrease may not be due to the ambiguity of the data. The strength $G_{\mathrm{m}}$ is a monotonically

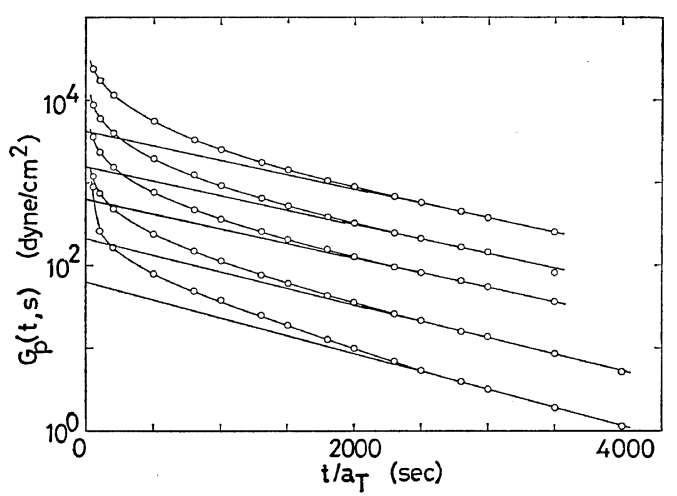

Figure 5. Examples of application of procedure $X$ for data of Figure 3. Shear strains $s$ are 0.34 , $3.41,6.83,13.7$, and 25.6 from top to bottom, respectively.

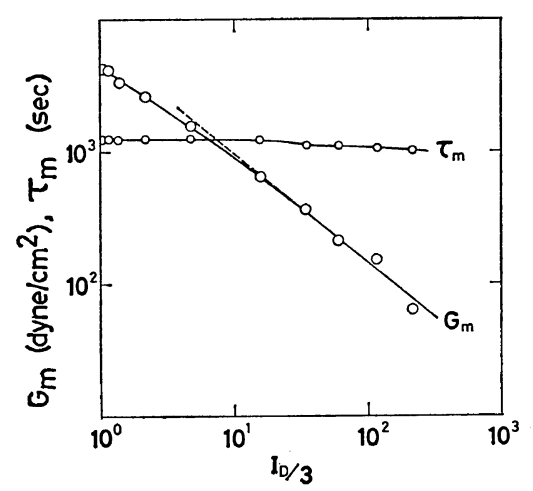

Figure 6. Longest relaxation time $\tau_{\mathrm{m}}$ and corresponding relaxation strength $G_{\mathrm{m}}$ plotted against $1 / 3$ of first invariant of strain $I_{D}$ for strain-dependent relaxation moduli of Figure 3. Dashed line is drawn with slope -0.83 .

decreasing function of $I_{D}$. The straight line in the range of large $I_{D}$ is drawn with the slope -0.83 , which was determined from the dependence of $\log G(t) \mid G(t, s)$ on $I_{D}$. The data for $G_{\mathrm{m}}$ lie on this line when $I_{D}$ is large. As the strain decreases, the dependence of $G_{\mathrm{m}}$ on $I_{D}$ becomes weaker and the data points deviate from the straight line of slope -0.83 . It seems possible to evaluate the $G_{\mathrm{m}}$ value at the limit of $s \rightarrow 0$ or $I_{D} / 3 \rightarrow 1$ in Figure 6 . The $G_{\mathrm{m}}$ values for small strains $(s \leqq 0.68)$ are within $10 \%$ of the limiting value so that they may be approximately regarded as the value for linear viscoelasticity.

\section{Concluding Remarks}

Figure 4 indicates that the relaxation strength decreases with increasing strain in a limited range of long times. This decrease can be related to the decrease in number of the effective entanglement couplings due to the large strain. Since entanglement coupling is supposed to be responsible for the relaxation mechanisms in the whole range of time scale studied here, the result may imply that there are two types of relaxation mechanisms caused by entanglement coupling. As reported earlier, the strengths of relaxation mechanisms for the longest one or few relaxation times are proportional to the third or higher power of the polymer concentration when $s \rightarrow 0$ while those for the plateau region are proportional to the second power. ${ }^{6}$ Region 3 of 
Figure 4 may correspond to those relaxation mechanisms for which the effect of varying concentration is larger. Studies are in progress to examine the effect of varying concentration and molecular weight on the relaxation mechanism of the longest relaxation time.

Another point of interest may be the possible use of the relaxation moduli obtained under large strain to predict stresses corresponding to other types of strain. We will discuss this problem in the following paper.

Acknowledgment. We are obliged to Iwamoto Seisakusho Co., Ltd., for the kind help in constructing the relaxometer.

\section{REFERENCES}

1. See for example, T. W. Spriggs, J. D. Huppler, and R. B. Bird, Trans. Soc. Rheology, 10, 191 (1966); M. Yamamoto, "Buttai no Henkeigaku", Seibundo-Shinkosha, Tokyo, 1972; M. Kurata, Zairyo, 21, 360 (1972).
2. See for example, A.V. Tobolsky, "Properties and Structure of Polymers," John Wiley \& Sons, Inc., New York, N.Y., 1960; J. D. Ferry, "Viscoelastic Properties of Polymers," 2nd ed., John Wiley \& Sons, Inc., New York-LondonSydney-Toronto, 1970.

3. L. J. Zapas and T. Craft, J. Res. Natl. Bur. Stand., A, 69, 541 (1965).

4. M. Tamura, M. Kurata, K. Osaki, Y. Einaga, and S. Kimura, Bull. Inst. Chem. Res. Kyoto Univ., 49, 43 (1971).

5. Y. Einaga, K. Osaki, M. Kurata, S. Kimura, and M. Tamura, Polymer J., 2, 550 (1971).

6. Y. Einaga, K. Osaki, M. Kurata, and M. Tamura, Macromolecules, 4, 87 (1971).

7. Y. Einaga, K. Osaki, M. Kurata, and M. Tamura, ibid., 5, 635 (1972).

8. M. Yamamoto, Zairyo, 21, 355 (1972).

9. J. D. Ferry and M. L. Williams, J. Colloid Sci., 7, 347 (1952).

10. A. V. Tobolsky and K. Murakami, J. Polymer Sci., 40, 443 (1959). 